\title{
Overall outcomes of laparoscopic-assisted ERCP after Roux-en-Y gastric bypass and sphincter of Oddi dysfunction subgroup analysis
}

\section{(ㄷ)(i) $\Theta$}

\author{
Authors \\ Institutions \\ 1 Lovelace Health System, Albuquerque, New Mexico, \\ United States \\ 2 Sanford Medical Center, Fargo, North Dakota, United \\ States \\ 3 Geisinger Medical Center, Danville, Pennsylvania, United \\ States
}

David May ${ }^{1}$, Ellen Vogels², David Parker ${ }^{3}$, Anthony Petrick ${ }^{3}$, David Diehl ${ }^{3}$, Jon Gabrielsen ${ }^{3}$ submitted 8.8 .2018

accepted after revision 4.12 .2018

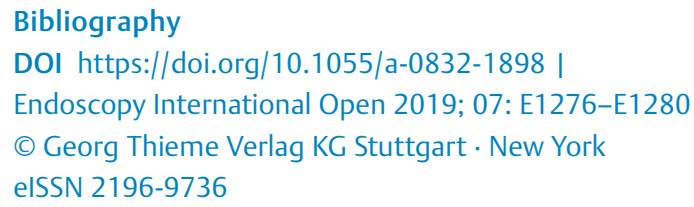

Corresponding author

Jon Gabrielsen, MD, FACS, FASMBS, Division of Bariatric and Foregut Surgery, Geisinger Medical Center, 100 North Academy Avenue, Danville, PA 17821

Fax: +1-570-271-5785

Jdgabrielsen@geisinger.edu

\section{ABSTRACT}

Background and study aims Biliary access following Roux-en-Y gastric bypass (RYGB) anatomy presents a significant challenge. Long-term outcomes of laparoscopic-assisted trans-gastric ERCP (LA-ERCP) including sphincter of
Oddi dysfunction (SOD) subtypes have not been thoroughly examined. Our study aims to present our overall outcomes of trans-gastric LAERCP and examine a significant subgroup of patients with SOD after RYGB.

Patients and methods A retrospective review of RYGB patients who underwent LA-ERCP between 2009 and 2016 identified 51 patients. A subgroup of 22 patients with SOD were examined and contacted by phone survey to determine long-term symptom resolution.

Results Post-procedure length of stay was 1.9 days (SD 3.0). There was one conversion from laparoscopic to open procedure. Selective cannulation rate was $100 \%$. Mean follow-up was 14.6 months. There were two major operative complications, two major ERCP-related complications, and five wound infections (9.8\%). No deaths or episodes of pancreatitis occurred. Seventeen patients had biliary SOD (Type $I=9$, Type $I I=8$ ). The remaining four had pancreatic SOD (Type I=1, Type $I=4$ ). SOD subgroup follow-up was 21.4 months (SD 18.1). All patients with Type I biliary and $75 \%$ with Type I pancreatic SOD reported complete resolution of their symptoms.

Conclusions Consistent with other published series, LAERCP yields excellent cannulation rates after RYGB. Successful treatment of pancreatic and Type 1 biliary SOD suggests that there is significant symptomatic benefit to treating this patient population. However, an overall complication rate of approximately $15 \%$ with LAERCP leaves open the possibility for improvements in access techniques in post-RYGB patients.

\section{Introduction}

Obesity and rapid weight loss are well established risk factors for development of cholelithiasis and both factors are relevant in the bariatric surgical population. The American Society for Metabolic and Bariatric Surgery (ASMBS) estimates that 196,000 bariatric procedures were performed in the United States in 2015, 23.1\% of which were Roux-en-Y gastric bypasses (RYGB), placing a large sector of the population at risk for gallstone disease [1].
Risk of gallstones after bariatric surgery varies by procedure and is significantly higher with RYGB compared to laparoscopic sleeve gastrectomy (LSG). In addition, the risk appears to be greatest in the first 6 months after bariatric surgery [2]. Data have been conflicting, however, with regard to development of symptomatic cholelithiasis after RYGB and the ASMBS does not currently recommend removal of normal or asymptomatic gallbladders [3].

Management of symptomatic cholelithiasis after bariatric surgery is generally straightforward; however, treatment of 
choledocholithiasis or sphincter of Oddi dysfunction (SO) are more complex due to the altered luminal anatomy associated with RYGB and other malabsorptive procedures which render the ampulla of Vater inaccessible by conventional techniques. Many approaches have been described to overcome the anatomic hurdles associated with accessing the ampulla of Vater and common bile duct after RYGB in particular. Modalities including percutaneous transhepatic cholangiography, balloonassisted enteroscopy (single and double), spiral enteroscopy, endoscopic ultrasound-guided stent endoscopic retrograde cholangiopancreatography (ERCP) access to the remnant via the gastric pouch, and laparoscopic-assisted ERCP (LA-ERCP) have all been employed in the setting of post-RYGB biliary tract pathology. Laparoscopic ERCP has a long track record and is highly successful. Schreiner et al published a retrospective review of their experience, which directly compared their outcomes with balloon enteroscopy-assisted ERCP (BEA-ERCP) with LA-ERCP specifically looking at cannulation rates, therapeutic success, length of hospital stay, complications and procedure duration. They found that the papilla was successfully cannulated in only $59 \%$ of BEA-ERCP, as opposed to $100 \%$ of LA-ERCP. Of the 13 patients with failed BEA-ERCP, 11 went on to have LA-ERCP with $100 \%$ therapeutic success. There was no statistically significant difference in complication rates. They did note that deep enteroscopy has a more acceptable rate of success and may be considered if the patient has a shorter bypass (Roux + ligament of Treitz to jejuno-jejunal anastomosis limb length $<150 \mathrm{~cm}$ ) [4].

Patients who have undergone RYGB may develop problematic upper abdominal pain somewhere in their postoperative course that requires surgical evaluation. When typical causes of pain such as marginal ulcer, symptomatic cholelithiasis, biliary dyskinesia, internal hernia, and others have been ruled out, choledocholithiasis and SOD must be considered. SOD is a rare disorder in both the bariatric and non-bariatric population but represents a relatively common indication for ERCP after RYGB and is in the differential diagnosis for post-RYGB upper abdominal pain, particularly if associated with liver function test or pancreatic enzyme elevations. However, there is little data in the RYGB population examining SOD treatment outcomes. Lim and associates reported on a group of 35 patients with suspected SOD after RYGB [5]. We sought to report our overall experience with LA- ERCP and also report the results of ERCP/sphincterotomy in our cohort of patients with suspected SOD.

\section{Patients and methods}

Using our prospectively maintained institutional database, we conducted a retrospective review of RYGB patients who underwent LA-ERCP between 2009 and 2016 and identified 51 patients. Records were reviewed for preoperative clinical characteristics, as well as perioperative and long-term outcomes. Data collected included patient age, body mass index (BMI), diagnosis, medical comorbidities, lab abnormalities, presence of biliary dilation, and acuity. Operative statistics were collected including case duration, estimated blood loss, conversion to open procedure and any complication. LA-ERCP was performed using four to five trocars, at least one of which was $12 \mathrm{~mm}$. Gastric access was obtained via a balloon-anchored $12-\mathrm{mm}$ port accommodating a diagnostic ERCP scope. The gastrotomies were closed with either an endoscopic stapler or interrupted sutures. Betadine was used to cleanse the trans-gastric port incision site. Rectal Indomethacin was selectively used for highrisk patients (Age $<30$, all females, pancreatic duct instrumentation, use of needle knife). Postoperative data collected included hospital length of stay, resolution of symptoms, complications (surgical, cardiac, pulmonary, renal, urinary, etc.), wound complications, infections, blood product transfusions, emergency department (ED) visits and hospital readmissions. Patients with SOD were classified into biliary and pancreatic subtypes according to the Milwaukee classification system [6]. A subgroup of 22 patients with SOD was examined and contacted by phone survey to determine long term symptom resolution.

\section{Results}

Fifty-one patients underwent LA-ERCP at our institution between 2009 and 2016. Average post-procedure length of hospital stay was 1.9 days (SD 3.0). The study population was $88 \%$ female (45) with a mean age of 55.4 years (SD 10.9) and BMI of $32.2 \mathrm{~kg} / \mathrm{m} 2$ (SD 9.3) at the time of LA-ERCP. Comorbidities included Type 1 diabetes in four (8\%), Type 2 diabetes in 14 $(27 \%)$, coronary artery disease in seven (14\%), renal disease in four $(8 \%)$, obstructive pulmonary disease in one $(2 \%)$, hypertension in 25 (49\%), and venous thromboembolism in eight patients (16\%) (\ Table 1). Forty-eight patients (94\%) had "biliary-type" pain preoperatively.

ERCP was indicated for choledocholithiasis (24 patients, $47.1 \%$ ), SOD in 22 patients, $43.1 \%$ ), bile duct stricture in three patients, $5.9 \%$ ) and postoperative bile leak in one patient, $2.0 \%)$. One procedure $(2.0 \%)$ was done for suspected malignancy but no biliary pathology was identified ( $\triangleright$ Table 1 ). The average duration of the procedure was 3.1 hours (SD 1.3) with one conversion ( $2 \%$ from laparoscopic to open. Selective cannulation rate was $100 \%$. Mean follow-up with surgical or gastroenterology clinic was 14.6 months (SD 17.6). There were no perioperative mortalities and no cases of clinically significant pancreatitis. There were six readmissions (12\%) within 30 days of the procedure and seven patients (13.7\%) required evaluation in the Emergency Department within 30 days. The total 90 day complication rate was $15.7 \%(n=8)$. There were three major complications (6\%) which included an organ space infection requiring radiologic-guided drainage, acute blood loss requiring blood transfusion, and a patient with a dislodged gastrostomy tube and obstructive hemobilia requiring reoperation and repeat ERCP. There were five minor complications which included four surgical site infections (SSI) requiring antibiotics alone ( 2 superficial, 2 organ space) and one readmission for cholangitis due to suspected obstructed externalized biliary drain treated with antibiotics and percutaneous drain removal ( $>$ Table 2 ). There was one death documented within 6 months of the procedure which was due to progression of primary sclerosing cholangitis and unrelated to the procedure. 
- Table 1 Patient demographics.

\begin{tabular}{|l|c|}
\hline Variable & $\mathbf{N}=\mathbf{5 1}$ \\
\hline Age, years, mean (SD) & $55.4(10.9)$ \\
\hline Gender, female & $45(88 \%)$ \\
\hline BMI, mean (SD) & $32.2(9.3)$ \\
\hline Elective & $27(53 \%)$ \\
\hline Comorbidities & \\
\hline - Type I DM & $4(8 \%)$ \\
\hline - Type II DM & $14(27 \%)$ \\
\hline - CAD & $7(14 \%)$ \\
\hline - CKD & $4(8 \%)$ \\
\hline - COPD & $1(2 \%)$ \\
\hline - HTN & $25(49 \%)$ \\
\hline - VTE & $8(16 \%)$ \\
\hline Diagnosis & \\
\hline - Choledocholithiasis & $24(47.1 \%)$ \\
\hline - SOD & $22(43.1 \%)$ \\
\hline - Bile leak & $1(2.0 \%)$ \\
\hline - Bile duct stricture & $3(5.9 \%)$ \\
\hline - No pathology identified & $1(2.0 \%)$ \\
\hline bolism; sonary artery disease; CKD, chronic kidney disease; COPD, chronic ob- \\
\hline
\end{tabular}

Of the 22 patients with SOD, 17 (77\%) had biliary SOD and five $(23 \%)$ had pancreatic SOD. In the biliary SOD group, nine (41\%) were Type 1 and eight (36\%) were Type 2 and in the pancreatic SOD group one had (5\%) Type 1 and four (18\%) Type 2 according to the Milwaukee classification system. Follow-up of this SOD subgroup included telephone contact and was 21.4 months (SD 18.1). Overall, 16 patients (72.7\%) reported complete symptom resolution with six $(27.3 \%)$ reporting no improvement in symptoms. All patients with biliary Type $1 \mathrm{SOD}$ and pancreatic Type 1 SOD reported complete resolution of their symptoms. Type II biliary and Type II pancreatic SOD both had less reliable symptom relief with five $(62.5 \%)$ and one patient $(25 \%)$ reporting no change in their pain respectively. There was one patient with type II pancreatic SOD who continued to struggle with ongoing episodes of recurrent pancreatitis ( Table 3).

\section{Discussion}

Consistent with other published series, our series confirms that LA-ERCP can be safe with nearly $100 \%$ cannulation rates after RYGB. In comparison, pure endoscopic approaches have shown much lower cannulation rates with Schreiner et al reporting $59 \%$ successful cannulation with BEA-ERCP and Desai

\begin{tabular}{|l|c|}
\hline Table 2 Outcomes. & $51(100)$ \\
\hline Cannulation rate, $\mathrm{n}(\%)$ & $3.1(1.3)$ \\
\hline Operative time, hours, mean (SD) & $1(2)$ \\
\hline Conversion to open, $\mathrm{n}(\%)$ & $1.9(3.0)$ \\
\hline Length of stay, days, mean (SD) & $14.6(17.6)$ \\
\hline Length of follow-up, months, mean (SD) & $6(12)$ \\
\hline Readmission within 30 days, $\mathrm{n}(\%)$ & $8(15.7)$ \\
\hline Complications, Total, $\mathrm{n}(\%)$ & $5(9.8)$ \\
\hline - Minor, $\mathrm{n}(\%)$ & $3(5.9 \%)$ \\
\hline - Major, $\mathrm{n}(\%)$ & \\
\hline
\end{tabular}

- Table 3 Sphincter of Oddi dysfunction outcomes.

\begin{tabular}{|c|c|c|c|}
\hline \multirow{2}{*}{} & \multicolumn{3}{|c|}{ Symptom resolution } \\
\hline & $\mathbf{N}=\mathbf{2 2}$ & Complete & No change \\
\hline Biliary SOD & $17(77 \%)$ & $12(70.6 \%)$ & $5(29.4 \%)$ \\
\hline - Type 1 & $9(41 \%)$ & $9(100 \%)$ & 0 \\
\hline - Type 2 & $8(36 \%)$ & $3(37.5 \%)$ & $5(62.5 \%)$ \\
\hline Pancreatic SOD & $5(23 \%)$ & $4(80 \%)$ & $1(20 \%)$ \\
\hline - Type 1 & $1(5 \%)$ & $1(100 \%)$ & 0 \\
\hline - Type 2 & $4(18 \%)$ & $3(75 \%)$ & $1(25 \%)$ \\
\hline SOD, sphincter of Oddi dysfunction & & \\
\hline
\end{tabular}

et al reporting $57 \%$ with spiral enteroscopy [7]. Schreiner went on to examine the relationship of successful cannulation with depth of enteroscopy. After reviewing RYGB operative reports for 20 of their patients, they found that their success rate withr BEA-ERCP in patients with a Roux + ligament of Treitz to jejunojejunal anastomosis (LTJJ) limb length $<150 \mathrm{~cm}$ was $88 \%$. The success rate dropped precipitously when total length exceeded $150 \mathrm{~cm}$. Patients with a Roux + LTJJ limb length of 150 to $225 \mathrm{~cm}$ had a cannulation rate of $33 \%$. The success rate dropped to $0 \%$ in lengths $>225 \mathrm{~cm}$ [4]. This is directly relevant to our patient population because at our institution, it is our standard practice to create a $150-\mathrm{cm}$ Roux limb and $50-\mathrm{cm}$ biliopancreatic (or LTJJ) limb when performing RYGB for obesity and related diseases. An endoscopy-first approach would result in an estimated two-thirds of patients needing a second procedure for successful treatment. As hospital systems continue to examine utilization and length of stay to improve cost effectiveness and efficiency, a primary intervention with such a low success rate will become increasing difficult to justify. The tradeoff for abandoning an enteroscopy-assisted first approach is likely a higher complication rate associated with an operative approach, which introduces the risk of surgical site infections (SSI), hernia, enteric leak, and hemorrhage. In our series, only two complications ( 1 major and 1 minor) were directly attributed to ERCP, whereas the remaining seven complications were related to 
surgical access ( 3 major and 4 minor). Our overall complication rate of $15.7 \%$ is comparable to the findings of Grimes et al who reported a rate of $14 \%$ (6/42) in their LA-ERCP subgroup. Unfortunately, that series also included a large number of percutaneous ERCP via mature gastrointestinal-tube tracts preventing further comparison [8].

In our study, the majority of postoperative complications were SSIs with a rate of $9.8 \%(n=5)$, which reflects the challenge of maintaining sterility when the gastrointestinal tract is entered. Published rates for SSI in clean-contaminated cases range from $3.3 \%$ to $3.94 \%$ based on American College of Surgeons National Surgical Quality Improvement Program data [9]. We use a 15-mm balloon port placed directly into the gastric remnant for endoscopic access and then over-drape the remainder of the operative field to maintain sterility during the endoscopic portion of the procedure. Unfortunately, the balloon port must then be removed, exposing the patient to seeding of enteric bacteria along the trocar entry point. This likely accounts for an SSI rate that is considerably higher than that expected with a clean-contaminated procedure. Further examination of our operative technique is warranted to identify methods to lower the incidence of SSI in LA-ERCP. Proposed changes include the use of a wound protector at the gastric trocar site, intracorporeal cleansing of the soiled trocar with betadine or Dakin's prior to removal, or packing of the gastric trocar wound.

The clear majority of complications in this series were related to the laparoscopic access portion of the procedure. ERCPrelated complications were low with one major and one minor complication. One of these was a patient who developed obstructive hemobilia and required repeat ERCP for clot removal. The other patient had undergone ERCP and stenting for postcholecystectomy bile leak. A biliary drainage catheter was left in place for decompression and brought out percutaneously via the gastric remnant, which had been secured to the abdominal wall. The patient was readmitted with cholangitis after noting several days of decreased drainage. Antibiotic treatment was initiated, a cholangiogram was performed showing resolution of the previously seen cystic duct leak, and the drain was removed with rapid improvement in symptoms.

The overall complication rates of approximately $15 \%$ in our series and in that of Grimes show that LA-ERCP is not without downside and there is certainly room for techniques that would be less invasive with an equally high success rate. More recently, endoscopic ultrasound-directed trans-gastric ERCP (EDGE) has been developed as a novel endoscopic technique that holds promise to gain access to the ampulla in patients whose anatomy would render it otherwise largely inaccessible. This technique does hold some promise and in a retrospective study comparing LA-ERCP to EDGE, technical success rates were similar with similar overall adverse event rates (18\% LA-ERCP vs. $21 \%$ EDGE). Of note, total procedure time was significantly shorter with EDGE ( $79 \mathrm{~min}$ vs. $183.5 \mathrm{~min}$ ) as was hospital stay ( 0.7 days vs. 2.65 days) [10]. The ultimate role of EDGE in management of post-RYGB biliary tract disease is continuing to evolve. Our current practice is to perform LA-ERCP in patients where cholecystectomy is indicated or when the patient has had a laparoscopic RYGB and no other significant intra-abdominal operations, particularly when they were performed open. EDGE has had an increasing role at our institution, particularly in patients with an anticipated hostile abdomen, or in patients where repeat access to the biliary tree will be required (i.e. stent removal). Enteroscopy plays a minor role at our institution and is often attempted because of the long roux limb lengths ( $150 \mathrm{~cm}$ or greater) created by our surgeons when performing the procedure.

Post-gastric bypass patients with unexplained abdominal pain can be a very challenging group in terms of identifying and treating the underlying cause of the pain. In our practice, patients with abdominal pain after RYGB will be worked up with upper endoscopy, Upper gastrointestinal series, complete blood count, LFT, computed tomography scan if the previous studies were negative, and ultrasound of the gallbladder if present. In some cases, a hepatobiliary iminodiacetic acid scan will be obtained looking for reflux of the tracer into the gastric remnant, as significant bile reflux gastritis can be a cause of chronic upper abdominal pain in post-RYGB patients. If all these tests are negative, patients will generally undergo a diagnostic laparoscopy and remnant endoscopy. It is this last group of patients (in whom all other studies have been negative) and who have a history at least somewhat consistent with biliary type pain, abnormal LFTs, abnormal pancreatic enzymes, or a combination of those things in whom we consider as potentially having the diagnosis of SOD. In those instances, we offer laparoscopy with remnant endoscopy and ERCP. SOD is thus mainly considered after all other reasonable etiologies have been ruled out with a thorough work-up.

Our success in symptom improvement for biliary Type 1 SOD and pancreatic Type 1 SOD suggests that there is reliable benefit in treating this patient population with an acceptable risk profile. Our findings align with multiple previously published studies in the non-bariatric surgical population, which have shown the best result in Type 1 SOD and a lower chance of symptomatic improvement in Types 2 and 3 SOD (biliary or pancreatic), suggesting that the disorder and its treatment are not fundamentally different between patients who have undergone bariatric surgery and those who have not. This stands in contrast to the results published by Lim et al., where the response in post-RYGB patients with type II SOD was significantly higher than those with type I [5].

In our practice, patients with post-cholecystectomy biliarytype pain without radiographic or laboratory abnormalities (Type III SOD) are not offered LA-ERCP. The EPISOD trial demonstrated with a high level of evidence that this group of patients are likely better treated with alternative modalities [11]. Although we are limited by sample size, our data also suggest significantly less benefit for those patients presenting with biliary SOD type 2 , which is also consistent with published series that demonstrate a sequential decrease in symptom resolution after treatment of SOD types 2 and 3. Previous studies have shown the best results in Type 2 SOD patients with documented elevation of SOM pressure [12]. However, our gastroenterologists do not perform sphincter of Oddi manometry in these patients. 
It is important to note that our study is limited by lack of a standardized measure for treatment success. We rely exclusively on patient reporting of symptom improvement, and we have not yet implemented any a validated survey for pain or quality of life in this population.

\section{Conclusions}

Consistent with other published series, excellent cannulation rates can be obtained with LA-ERCP after RYGB. SSIs account for most of the access-related complications with this approach. While LA-ERCP is highly effective and is the current gold standard from a technical success standpoint, the overall complication rate of approximately $15 \%$ shows that there is room for emerging techniques, such as EDGE, that may offer equivalent technical success with other potential advantages vs. LA-ERCP.

Regarding management of SOD in the bariatric surgery population, our results suggest that response to treatment for different subtypes of SOD in a post-RYGB population appears to be consistent with those previously published in the non-bariatric population. Treatment strategies for dealing with postRYGB pain and suspected SOD should follow the same approach taken in the non-bariatric population.

\section{Competing interests}

None

\section{References}

[1] Estimate of Bariatric Surgery Numbers, 2011-2016. Available at: https://asmbs.org/resources/estimate-of-bariatric-surgery-numbers [Accessed January 8, 2019]
[2] Tsirline VB, Keilani ZM, El Djouzi S et al. How frequently and when do patients undergo cholecystectomy after bariatric surgery? Surg Obes Relat Dis Off J Am Soc Bariatr Surg 2014; 10: 313-321

[3] American Society for Metabolic and Bariatric Surgery. Available at: http://www.choosingwisely.org/societies/american-society-for-metabolic-and-bariatric-surgery/ [Accessed January 8, 2019]

[4] Schreiner MA, Chang L, Gluck M et al. Laparoscopy-assisted versus balloon enteroscopy-assisted ERCP in bariatric post-Roux-en-Y gastric bypass patients. Gastrointest Endosc 2012; 75: 748 - 756

[5] Lim CH, Jahansouz C, Freeman ML et al. Outcomes of Endoscopic Retrograde Cholangiopancreatography (ERCP) and Sphincterotomy for Suspected Sphincter of Oddi Dysfunction (SOD) Post Roux-En-Y Gastric Bypass. Obes Surg 2017; 27: 2656- 2662

[6] Feldman M, Friedman LS, Brandt L] (Hrsg) Biliary tract motor function and dysfunction. In: Sleisenger and Fordtran's gastrointestinal and liver disease: pathophysiology/diagnosis/management. Philadelphia, PA: Saunders/Elsevier; 2016: 1078 - 1084

[7] Desai SV, Naveed M, Jazwinski A et al. Spiral enteroscopy versus laparoscopic-assisted endoscopy for completion of ERCP in patients with Roux-en-Y gastric bypass surgery. Gastrointest Endosc 2011; 73: AB122

[8] Grimes KL, Maciel VH, Mata W et al. Complications of laparoscopic transgastric ERCP in patients with Roux-en-Y gastric bypass. Surg Endosc 2015; 29: 1753-1759

[9] Ortega G, Rhee DS, Papandria D] et al. An evaluation of surgical site infections by wound classification system using the ACS-NSQIP. J Surg Res 2012; 174: $33-38$

[10] Kedia P, Tarnasky PR, Nieto J et al. EUS-directed transgastric ERCP (EDGE) versus laparoscopy-assisted ERCP (LA-ERCP) for Roux-en-Y gastric bypass (RYGB) anatomy: a multicenter early comparative experience of clinical outcomes. J Clin Gastroenterol 2019; 53: 304308

[11] Cotton PB, Durkalski V, Romagnuolo J et al. Effect of endoscopic sphincterotomy for suspected sphincter of Oddi dysfunction on painrelated disability following cholecystectomy: the EPISOD randomized clinical trial. JAMA 2014; 311: $2101-2109$

[12] Small AJ, Kozarek RA. Sphincter of Oddi dysfunction. Gastrointest Endosc Clin N Am 2015; 25: 749-763 